\title{
Afghanistan: Ungewisse Zukunft der Transition
}

\author{
Marco Overhaus und Michael Paul*
}

\begin{abstract}
The road map for the transition of security responsibilities from foreign to Afghan security forces was once more confirmed during the NATO summit in Chicago in May 2012. While this road map is very likely to be implemented on schedule, not least for domestic political reasons in ISAF-nations, the prospects for transition to become "irreversible" remain uncertain. The main external actors of the transition, most notably the US and other ISAF-countries, will not only have to engage in longer-term support for building the Afghan security sector. Even more importantly, they will have to solve those contradictions which have plagued their policies since the fall of the Taliban.
\end{abstract}

Keywords: Afghanistan, international actors, security sector, transition

Afghanistan, internationale Akteure, Sicherheitssektor, Transition

\section{Einleitung}

$\mathrm{B}$ is Mitte 2013 werden einheimische Polizei und Armee in ganz Afghanistan die Führung bei der Gewährleistung von Sicherheit übernehmen. Der Prozess zur Übergabe der Sicherheitsverantwortung (Transition) von den ausländischen auf die afghanischen Kräfte soll bis Ende 2014 vollständig abgeschlossen sein. Das Mandat der Internationalen Afghanistan-Schutztruppe (ISAF) wird dann beendet und die ausländische Militärpräsenz in eine deutlich kleinere Unterstützungs- und Ausbildungsmission ohne Kampfauftrag überführt.

Es gibt wenig Zweifel daran, dass dieser, zuletzt im Mai 2012 während des NATO-Gipfels in Chicago verkündete Fahrplan eingehalten wird. Zu groß ist der innenpolitische Druck in den ISAF-Ländern, das militärische Afghanistan-Engagement zu beenden oder zumindest deutlich zu reduzieren.

Die entscheidende Frage lautet aber, inwiefern die Transition tatsächlich dauerhaft und „unumkehrbar" sein wird, so wie sich dies die afghanische Regierung, die Truppensteller der ISAF und die internationalen Geber als Ziel gesetzt haben. ${ }^{1}$ Es geht darum, langfristig Sicherheit für Afghanistan zu schaffen und dabei den Grundstein für eine friedliche Entwicklung in dem Land zu legen.

Die wirtschaftlichen und politischen Rahmenbedingungen der Transition sind mehr als ungünstig, sie lassen sich zudem kurzund mittelfristig nur noch schwer von außen beeinflussen: Afghanistan bleibt auf absehbare Zeit stark von internationalen Hilfen abhängig, eine konstruktive Rolle der Nachbarstaaten insbesondere Pakistans - ist nicht in Sicht. Der politische Prozess zwischen afghanischer Regierung und den regierungsfeindlichen Gruppen kommt bisher nicht in Gang.

* Dr. Marco Overhaus ist wissenschaftlicher Mitarbeiter der Forschungsgruppe EU-Außenbeziehungen, Dr. Michael Paul ist wissenschaftlicher Mitarbeiter der Forschungsgruppe Sicherheitspolitik der Stiftung Wissenschaft und Politik.

1 Vgl. Afghanistan and the International Community. From Transition to the Transformation Decade, Conference Conclusions, Bonn, Dezember 2011, http://www.auswaertiges-amt.de/cae/servlet/contentblob/603684/ publicationFile/162776/Conference Conclusions - International Afghanistan_Conference_Bonn_2011_engl.pdf (10. Juli 2012) sowie Chicago Summit Declaration on Afghanistan, Chicago, 21. Mai 2012, http://www.nato. $\mathrm{int} / \mathrm{cps} / \mathrm{en} /$ natolive/official_texts_87595.htm, (10. Juli 2012).
Angesichts dieser Lage sollten sich die treibenden ausländischen Akteure der Transition - in erster Linie die USA und ihre ISAF-Partner - in den kommenden Jahren darauf konzentrieren, die bisherigen Widersprüche ihrer Afghanistan-Politik so weit wie möglich abzubauen. Der Versuch, die Gewährleistung von Sicherheit auch umfassend, und nicht bloß im Sinne eines quantitativen Aufwuchses der Afghan National Security Forces (ANSF), in afghanische Verantwortung zu überführen, wurde frühestens seit 2009 ernsthaft unternommen. Gerade deshalb darf die verbreitete Ernüchterung über ausbleibende Erfolge in Afghanistan nicht zu einem vorschnellen Erlahmen des Engagements führen.

\section{Schwierige wirtschaftliche und politische Rahmenbedingungen}

Die ökonomischen und politischen Rahmenbedingungen stellen für den langfristigen Erfolg der Transition eine enorme Herausforderung dar. Afghanistan gehört zu den am wenigsten entwickelten Ländern der Erde, gemessen am Bruttoinlandsprodukt pro Kopf zählt es sogar zu den zehn ärmsten Staaten. Drei Viertel der Bevölkerung können weder lesen noch schreiben. ${ }^{2}$

Landwirtschaft und Bergbau könnten sich in den kommenden Jahren zu Treibern wirtschaftlichen Wachstums auch unabhängig von ausländischen Hilfen entwickeln. Darüber hinaus ist es dem afghanischen Staat durch Reformen gelungen, die eigenen Steuer- und Zolleinnahmen zu erhöhen. ${ }^{3}$ Gleichzeitig werden jedoch die operativen Ausgaben noch stärker als die Einnahmen steigen. Grund ist der notwendige Finanzierungsbedarf für die afghanischen Sicherheitskräfte sowie für den Erhalt und die Fortführung zahlreicher Entwicklungsprojekte, die von internationalen Gebern angestoßen wurden. Afghanistan wird daher auch auf längere Sicht von internationalen Hilfsleistungen abhängig bleiben. ${ }^{4}$ Hinzu kommt die Bedeu-

2 Vgl. The World Bank, Afghanistan: From Transition to Transformation Briefing for the Tokyo Conference, Washington D.C., 1. Juli 2012, S. 3, http://siteresources.worldbank.org/SOUTHASIAEXT/Resources/ 223546-1328913542665/8436738-1341156360475/Afghanistan-TransitionTransformation-2012-English.pdf, (10. Juli 2012).

3 The World Bank, Afghanistan economic update, Washington D.C., Oktober 2011, S.7.

4 Ebd., S. 9 . 
tung der „Kriegswirtschaft“, die durch die massive Präsenz ausländischer Soldaten entstanden ist. Vor diesem Hintergrund ist absehbar, dass die Übergabe der Sicherheitsverantwortung zunächst zu einer Abschwächung der afghanischen Wirtschaftskraft führen wird.

Auch die politischen Entwicklungen in Afghanistan bieten bisher wenig Anlass zu Optimismus. Seit 2011 bemühen sich insbesondere die USA verstärkt um einen politischen Dialog mit den Aufständischen. Dazu zählen grundsätzlich alle relevanten Gruppen wie die Hezb-e Islami, das Haqqani-Netzwerk und die Taliban unter Führung von Mullah Omar. Im Mittelpunkt steht jedoch bislang der Versuch, mit den Taliban über die Eröffnung eines Verbindungsbüros in Katar ins Gespräch zu kommen, um einen politischen Prozess zu beginnen. Während weiterhin informelle Kontakte gemeldet werden, ist dieser Prozess von anhaltenden Problemen gekennzeichnet und wurde zuletzt im März 2012 von den Taliban suspendiert. ${ }^{5}$ Die Taliban sind seit 2001 zum Sammelbecken derjenigen Kräfte avanciert, die allgemein mit der herrschenden Situation unzufrieden sind; entsprechend heterogen sind auch ihre Ziele.

Die Hürden für politische Verhandlungen sind ohnehin groß: Bislang haben die Taliban wenig Interesse an direkten Gesprächen mit Vertretern der afghanischen Regierung gezeigt, weil sie diese nicht als legitime Vertretung Afghanistans anerkennen wollen. Auch ist fraglich, inwiefern die verschiedenen Insurgenten-Gruppen genügend politische Kohäsion aufbringen, um den Prozess zu einem Ergebnis zu führen, und in der Lage sein werden, dieses Ergebnis am Ende auch umzusetzen.

Die „Aussöhnung“ (reconciliation) hat sich zu einer tragenden Säule der amerikanischen Afghanistan-Politik und damit auch der Transition entwickelt. ${ }^{6}$ Die Teilnehmer der AfghanistanKonferenz, die im Dezember 2011 in Bonn stattfand, dokumentierten erstmals die Unterstützung aller internationalen Partner für Friedensverhandlungen, die möglichst inklusiv sein sollen. Die aufständischen Gruppen sollen dabei der Gewalt und dem internationalen Terrorismus abschwören und die afghanische Verfassung respektieren. ${ }^{7}$

Der Ausgang solcher Verhandlungen wird auch fundamentale Auswirkungen auf die zukünftige Rolle, den Umfang und die nötigen Qualitäten der afghanischen Sicherheitskräfte haben. Dabei darf jedoch nicht unterschlagen werden, dass ein politisches Abkommen mit den Taliban das Ziel der Schaffung „entpolitisierter“ und professioneller Sicherheitskräfte in noch weitere Ferne rücken kann, da sie ihre eigenen politischen Vorstellungen einzubringen versuchen werden und daher die vorhandene Fragmentierung noch weiter verstärken könnten.

5 Afghanistan's Taliban suspend peace talks with US, in: BBC News Asia, 15. März 2012.

6 US-Präsident Barack Obama bezeichnete die politische Aussöhnung zwischen afghanischer Regierung und den Aufständischen als eine von fünf Säulen der Strategie seiner Regierung „to complete our mission and end the war." Karen DeYoung, "As U.S.-Taliban talks stall, hope for political solution dims", in: Washington Post, 9. Mai 2012.

7 Council on Foreign Relations, Afghanistan and the International Community. From Transition to the Transformation Decade, Conference Conclusions, http:// www.cfr.org/afghanistan/conference-conclusions-international-conferenceafghanistan-december-2011/p26689.

\section{Die Entwicklung der afghanischen Sicherheitskräfte}

Im Jahr 2002, zu Beginn des internationalen Afghanistan-Engagements und nach dem Sturz der Taliban, sah der Ansatz zum Aufbau der afghanischen Sicherheitskräfte noch eine Arbeitsteilung vor, die die Verantwortung für die internationale Unterstützung beim Aufbau der afghanischen Nationalarmee (ANA) in die Hände der USA legte. Für die Unterstützung beim Aufbau der afghanischen Polizei übernahmen zunächst Deutschland und seit 2007 die Europäische Union die Führungsrolle. Diese Art der Arbeitsteilung erwies sich als wenig erfolgreich.

Die Bemühungen der USA zum Aufbau der afghanischen Armee zielten anfangs auf den Aufbau einer infanteristischen Hilfstruppe. ${ }^{8}$ Mit zunehmender Verschlechterung der Sicherheitslage rückte der quantitative Aufwuchs der afghanischen Sicherheitskräfte immer mehr in den Vordergrund, was zu einer stetigen Ausweitung der jeweiligen Zielgrößen führte. Noch im Februar 2002 sollten ANA und die Afghan National Police (ANP) bis 2010 jeweils auf 62.000 Kräfte aufgebaut werden, im Januar 2010 lautete das Ziel bereits 171.600 für die ANA und 134.000 für die afghanische Polizei. Im Juni 2011 schließlich wurde die aktuelle Zielgröße - 195.000 afghanische Soldaten und 157.000 Polizisten bis Oktober 2012 - festgelegt. Die Konzentration auf hohe Aufwuchszahlen ging einher mit immer kürzeren Ausbildungszeiten für eine zunehmende Zahl von lese- und schreibunkundigen Rekruten.

Die afghanische Polizei wurde während der ersten Jahre des internationalen Afghanistan-Engagements gegenüber der ANA vernachlässigt. Sie erhielt weniger Training, Ausrüstung und Besoldung als die Armee. Eine Ausnahme bildete lediglich die Afghan National Civil Order Police (ANCOP), eine paramilitärische Einheit, die auch Teil der afghanischen Polizei ist. Sie konnte sich zu einer relativ effektiven Komponente innerhalb der ANP entwickeln, nicht zuletzt, weil sie besonders intensiv von der NATO trainiert wurde. ${ }^{9}$ Ebenso wie die afghanische Armee wurde die Polizei im Zuge der Verschlechterung der Sicherheitslage zunehmend als militärische Hilfstruppe im Kampf gegen die Aufständischen eingesetzt. ${ }^{10}$

Als besonders kontraproduktiv erwiesen sich die gegensätzlichen Ansätze zwischen den USA und den Europäern beim Aufbau der afghanischen Polizei. Während erstere vor allem paramilitärische Kräfte zur Bekämpfung der regierungsfeindlichen Kräfte im Sinn hatten, strebten Deutsche und Europäer nach dem Ideal ziviler Polizeikräfte, freilich ohne die dafür notwendigen Ressourcen bereitzustellen. Hinzu kam, dass sowohl bei Armee und Polizei der Aufbau essenzieller Unterstützungsfunktionen - etwa im Bereich der Logistik - in den ersten Jahren vernachlässigt wurde.

Die operativen Fähigkeiten der ANA haben in den vergangenen Jahren schrittweise zugenommen. Seit dem ersten größe-

8 Michael Paul, „Licht am Ende des Tunnels? Der Aufbau der Afghanischen Nationalarmee“, in: Sicherheit und Frieden, 28 (2010) 1, S. 42-48.

9 US Department of Defense, Report on Progress toward Security and Stability in Afghanistan, Washington D.C., Oktober 2011, S. 35

10 Cornelius Friesendorf/Jörg Krempel, Militarized versus Civilian Policing: Problems of Reforming the Afghan National Police, Frankfurt am Main: Hessische Stiftung Friedens- und Konfliktforschung, 2011, S. 30 (PRIF-Report, 102). 
ren Einsatz im Juli 2003 finden zunehmend Operationen unter afghanischer Führung statt. Derzeit erfolgen 90 Prozent aller Einsätze gemeinsam mit der ISAF, davon über 40 Prozent unter afghanischer Führung. ${ }^{11}$ Die ANA ist in der Lage, Operationen bis zur Kompanieebene (etwa 120 Mann) selbst zu leiten. Allerdings kämpft in Afghanistan bisher kein einziges afghanisches Bataillon (Kandak) - als nächstgrößere militärische Einheit ohne die Unterstützung durch amerikanische Soldaten oder ISAF-Truppen. Selbst wenn manche Einheiten als fähig zum „unabhängigen“ Operieren eingestuft werden, bleiben auch diese auf Hilfe bei Wartung, Logistik und Sanitätswesen angewiesen. ${ }^{12}$

Bei der Polizei wurde noch im August 2011 keine von insgesamt 218 bewerteten Polizeieinheiten als „unabhängig“ einsatzfähig eingestuft, ${ }^{13}$ im Januar 2012 waren es immerhin schon 39 von 435 bewerteten Einheiten, also etwa neun Prozent. ${ }^{14}$

Die weitaus größte Herausforderung beim Aufbau der afghanischen Polizei ist deren Neuausrichtung: weg von der Aufstandsbekämpfung hin zur zivilen Polizeiarbeit. Darunter fallen die Bekämpfung der Kriminalität, die Schlichtung von Streitigkeiten, der enge Kontakt mit der Bevölkerung und die Zusammenarbeit mit der afghanischen Justiz. Die afghanische Justiz befindet sich weiterhin in einem desolaten Zustand. Bis 2012 wurden zudem keine Daten erfasst, um die zivile Polizeiarbeit in Afghanistan bewerten zu können. ${ }^{15}$

Afghanische Regierung und internationale Geber haben gerade erst damit begonnen, den zivilen Charakter der ANP stärker in den Vordergrund zu stellen und sie dabei systematischer mit der formellen sowie informellen afghanischen Justiz zu verknüpfen. ${ }^{16}$ Die europäische Polizeimission EUPOL-Afghanistan soll 2012 im Rahmen einer Revision des Mandats auf drei thematische Schwerpunkte konzentriert werden. Dies ist erstens die institutionelle Reform des afghanischen Innenministeriums, zweitens die Professionalisierung der ANP sowie drittens die Verknüpfung der Polizei- und Justizreform. ${ }^{17}$

Auch die qualitativen Aspekte der Fähigkeitsentwicklung bei den afghanischen Sicherheitskräften werden erst seit kurzer Zeit -

11 US Department of Defense, Report on Progress toward Security and Stability in Afghanistan. United States Plan for Sustaining the Afghanistan National Security Forces, Washington D.C., April 2012, S. 13.

12 Daten vom März 2012 weisen insgesamt 13 Kandaks als "unabhängig mit Beratern"aus, die Mehrheit der 156 evaluierten Verbände benötigt jedoch weiterhin Unterstützung. Vgl. Special Inspector General for Afghanistan Reconstruction (SIGAR), Quarterly Report to the United States Congress, 30. Januar 2012, S. 69; US Department of Defense, Report on Progress toward Security and Stability in Afghanistan. United States Plan for Sustaining the Afghanistan National Security Forces, Washington D.C., April 2012, S. 38 und 43.

13 Dabei wurde die Definition des Begriffs „unabhängig“ (independent) bereits gelockert, um der Tatsache gerecht zu werden, dass die meisten ANSF-Kräfte auch langfristig auf internationale Unterstützung angewiesen sein werden. Vgl. US Department of Defense, Report on Progress toward Security and Stability in Afghanistan, Washington, D.C., Oktober 2011, S. 43 und 45.

14 US Department of Defense, Report on Progress toward Security and Stability in Afghanistan. United States Plan for Sustaining the Afghanistan National Security Forces, Washington, D.C., April 2012, S. 51.

15 Erst ab Anfang 2012 sollten die „CUAT-Berichte“ (Commander's Unit Assessment Tool) spezifische Angaben zu dem Thema „community policing“ beinhalten. US Department of Defense, Report on Progress toward Security and Stability in Afghanistan, Washington, D.C., Oktober 2011, S. 42.

16 Anthony H. Cordesman, The Afghan War 10 Years on: Transition and the Coming Resource Crisis, Washington D.C.: Center for Strategic \& International Studies, November 2011, http://csis.org/files/publication/111011_Afghan_War_10_ years.pdf (8. März 2012), S. 6-7.

17 Bundesregierung, Fortschrittsbericht Afghanistan zur Unterrichtung des Deutschen Bundestages, Berlin: Presse- und Informationsamt der Bundesregierung, 2011, S. 26. zum Teil erst seit 2011 - von der ISAF in Angriff genommen. So konzentrieren sich ihre Trainings- und Unterstützungsaktivitäten nun auch verstärkt auf höherwertige Bereiche, wie insbesondere die Logistik, Führung und Einsatzunterstützung. ${ }^{18}$ Nach amerikanischer Einschätzung werden die ANSF in diesen kritischen Bereichen noch mindestens bis in das Jahr 2017 auf ausländische Unterstützung angewiesen bleiben.

Weitere wichtige Reformen betreffen die Angleichung der Gehälter für afghanische Polizisten an das Niveau der Armee sowie seit Oktober 2011 die Verlängerung der Grundausbildung für Polizeirekruten von sechs auf acht Wochen. Parallel dazu wurden die Trainingskapazitäten für die Polizei ausgebaut.

Während des NATO-Gipfels in Chicago wurde eine deutliche Reduzierung der afghanischen Sicherheitskräfte für die Zeit nach 2014 in Aussicht gestellt - von aktuell anvisierten 352.000 Soldaten und Polizisten auf dann nur noch 228.500. Eine sicherheitspolitische Begründung für diese neue Zahl wurde in Chicago nicht geliefert, stattdessen stand die Frage der zukünftigen Finanzierung der ANSF im Mittelpunkt. Die geschätzten Kosten für die reduzierte Truppe belaufen sich auf 4,1 Milliarden US-Dollar pro Jahr.

Eine derart starke Reduzierung der Sicherheitskräfte wird den weiteren Rückgriff auf Demobilisierungs- und Reintegrationsprogramme notwendig machen. In der Vergangenheit waren Ansätze zur Entwaffnung und Demobilisierung der zahlreichen Milizen allerdings erfolglos: Innerafghanische Widerstände und eine Strategie der NATO, die auf die Einbindung statt die Auflösung der Milizen setzte, sind dafür maßgeblich verantwortlich.

\section{Das Problem der Milizen}

Die ISAF hat sich mit dem Ziel der kurzfristigen Stabilisierung der Sicherheitslage immer wieder auf lokale Machthaber und deren Milizen gestützt. Damit hat sie zur Herausbildung ,alternativer Machtzentren "19 beigetragen, die wiederum auf längere Sicht das Risiko einer Entstaatlichung des Sicherheitsapparats bergen. In diesem Zusammenhang ist auch der Aufbau sogenannter lokaler Verteidigungskräfte (local defense forces) zu sehen, der seit 2006 und verstärkt seit 2009 vom US-amerikanischen Militär vorangetrieben wurde. ${ }^{20}$ Diese lokalen Kräfte sollen die Sicherheit dort gewährleisten, wo Polizei und Armee bislang noch nicht hinreichend präsent sind, also in erster Linie in den ländlichen Gebieten.

Zu den ersten Programmen dieser Art zählte die Afghan National Auxiliary Police (ANAP). Sie wurde 2006 im Süden Afghanistans lanciert und musste im April 2008 bereits wieder eingestellt werden. Die Hilfstruppe trug durch kriminelle Aktivitäten

18 James A. Schear/Wiliam B. Caldwell/Frank C. Digiovanni, »Developing Capacity for an Enduring Security Force«, in: PRISM, 2 (2011) 2, S. 135-144 (143); US Department of Defense, Report on Progress toward Security and Stability in Afghanistan, Washington, D.C., Oktober 2011, S. 38.

19 Thomas Ruttig, Afghanistan: Institutionen ohne Demokratie. Strukturelle Schwächen des Staatsaufbaus und Ansätze für eine politische Stabilisierung, Berlin: Stiftung Wissenschaft und Politik, Juni 2008 (SWP-Studie 17/2008), S. 13.

20 Philipp Münch, Strategielos in Afghanistan. Die Operationsführung der Bundeswehr im Rahmen der International Security Assistance Force, Berlin: Stiftung Wissenschaft und Politik, November 2011 (SWP-Studie 30/2011), S. 23. 
mehr zur Verbreitung von Unsicherheit als zur Herstellung von Sicherheit bei. Die Community Defense Initiative (CDI) wurde im Juli 2009 von amerikanischen Spezialkräften ins Leben gerufen und später in Local Defense Initiative (LDI) umbenannt. Im August 2010 wurden die einheimischen Kräfte der LDI in die Afghan Local Police (ALP) integriert.

Angehörige der lokalen Sicherheits- bzw. Verteidigungskräfte erfahren selbst im Vergleich zur afghanischen Polizei oder Armee nur eine rudimentäre Ausbildung, die im Falle ALP gerade einmal drei Wochen dauert. Darüber hinaus zeichnet sich ein Trend zur Verstetigung und Verfestigung lokaler Verteidigungsinitiativen ab, die sich somit zu einer Dauerlösung entwickeln könnten.

Internationale Nichtregierungsorganisationen wie Human Rights Watch beklagen, dass unzureichende Ausbildung, Ausstattung und Kontrolle der ALP und vergleichbarer Initiativen zu massiven Menschenrechtsverletzungen seitens dieser Kräfte geführt hätten. ${ }^{21}$ Sie fordern daher Maßnahmen zur besseren Auswahl, Ausbildung und Kontrolle der Mitglieder dieser staatlich sanktionierten Milizen. Zudem geht es darum, lokale Sicherheitskräfte wie die ALP besser in die formalen afghanischen Polizeistrukturen unter Kontrolle des Innenministeriums einzubinden. Schließlich ist auch der verstärkte Aufbau lokaler Regierungsinstitutionen von zentraler Bedeutung, um sicherzustellen, dass die afghanischen Sicherheitskräfte ebenso wie neu aufgebaute Dorfmilizen nicht von einzelnen Gruppen oder Machthabern, die eigene Interessen verfolgen, missbraucht werden.

\section{Afghanische Regierungsführung}

Funktionierende Verwaltungs- und Regierungsstrukturen in Afghanistan sind eine wesentliche Voraussetzung für den langfristigen Erfolg der Sicherheitstransition. Diese Strukturen haben einerseits eine unterstützende Funktion gegenüber den afghanischen Sicherheitskräften, vor allem in den Bereichen der Logistik, des Personalwesens, der Ausbildung und der Budgetverwaltung. Andererseits sollen Verwaltung und Regierung einen Beitrag zur Kontrolle der Sicherheitskräfte leisten.

Nach amerikanischen Einschätzungen ist das afghanische Verteidigungsministerium seit Oktober 2010 in der Lage, seine Aufgaben im Wesentlichen zu erfüllen, benötigt dabei jedoch weiterhin die Unterstützung der internationalen Koalition. ${ }^{22}$ Erklärtes Ziel ist es, dass bis Mitte 2014 alle Abteilungen des Ministeriums eigenständig arbeiten können. Dass dies ein sehr ambitioniertes Ziel ist, zeigt sich unter anderem daran, dass bis Anfang 2012 noch keine Abteilung im Verteidigungsministerium als fähig zum autonomen Handeln galt. ${ }^{23}$

21 Rachel Reid/Sahr Muhammedally, „Just Don't Call It a Militia“. Impunity, Militias, and the "Afghan Local Police“, New York: Human Rights Watch, September 2011, S. 8; Rebecca Barber, No Time to Lose. Promoting the Accountability of the Afghan National Security Forces, Oxford: Oxfam, Mai 2011, S. 8; Promoting the Accountability of the Afghan National Security Forces: Update, Oxford: Oxfam, September 2011, S. 3.

22 Dies entspricht der Kategorie "2B" im Bewertungssystem der Capability Milestones. US Department of Defense, Report on Progress toward Security and Stability in Afghanistan. United States Plan for Sustaining the Afghanistan National Security Forces, Washington, D.C., April 2012, S. 14.

23 Ebd., S. 16.
Das afghanische Innenministerium hinkt noch weiter zurück, es wurde Anfang 2012 wie bereits im Vorjahr unter Kategorie 3 der Capability Milestones eingestuft, benötigt also weiterhin erhebliche internationale Unterstützung. Dabei kann plausibel unterstellt werden, dass die von den USA bzw. der ISAF genutzten Bewertungssysteme tendenziell ein gegenüber der Realität zu positives Bild zeichnen, weil sie qualitative Indikatoren etwa zur Korruption - nicht angemessen berücksichtigen. ${ }^{24}$ Das Problem besteht jedoch nicht nur in den unzureichenden Kapazitäten in den Ministerien, sondern auch in der starken Politisierung dieser Strukturen, d.h. deren Missbrauch für politische und wirtschaftliche Partikularinteressen.

Bereits unmittelbar nach dem Sturz des Taliban-Regimes gewannen die Kommandeure der Nordallianz die Kontrolle über das bestehende Verteidigungsministerium und den Generalstab. ${ }^{25}$ Schlüsselpositionen wurden vor dem Hintergrund persönlicher und ethnischer Loyalitäten an ehemalige Kommandeure vergeben, nicht aufgrund von deren Befähigung. ${ }^{26}$

Politische Einflussnahme und Korruption bleiben auch im afghanischen Innenministerium zentrale Probleme. Eine im Sommer 2010 lancierte Arbeitsgruppe afghanischer und ausländischer Berater zur Reform des Innenministeriums kam zu dem Ergebnis, dass es insbesondere in zwei Bereichen unmittelbaren Handlungsbedarf gebe. Dies sei zum einen die Schaffung von Strukturen und Prozessen zur Kontrolle der afghanischen Polizei - etwa in Form einer Dienstaufsicht und eines verbindlich durchsetzbaren Verhaltenskodex. Zum anderen fehlt es an der funktionalen Trennung zwischen ministeriellen Aufgaben und der Durchführung von Polizeioperationen. Eine solche Trennung würde die politische Einflussnahme auf die Polizei und damit auch Korruption erschweren. ${ }^{27}$

ISAF-Truppensteller und andere internationale Akteure haben erst spät, zum Teil erst seit dem Ende der vergangenen Dekade, die Verbesserung der Regierungsführung und den Aufbau einer funktionierenden Verwaltung im Sicherheitssektor in den Fokus ihrer Unterstützung gerückt. ${ }^{28}$ Dabei gab es zwar durchaus Fortschritte, etwa bei der Ausbildung afghanischer Verwaltungskräfte, bei der Errichtung neuer Strukturen zur Bekämpfung der Korruption sowie bei den Reformen im Bereich der Militärjustiz. ${ }^{29}$ Dabei ist jedoch nicht immer klar, inwiefern institutionelle Reformen tatsächlich das zugrunde liegende Problem behoben haben. So ist beispielsweise der Aufbau der Militärjustiz nur ein Teilerfolg, weil das System für politische

24 Anthony H. Cordesman, Transition in the Afghanistan-Pakistan War: How Does This War End? Washington D.C.: Center for Strategic \& International Studies, Januar 2012, S. 98

25 Terence Kelly/Nora Bensahel/Olga Oliker, Security Force Assistance in Afghanistan: Identifying Lessons for Future Efforts, Santa Monica, Ca.: Rand, 2011 S. 27; International Crisis Group (ICG), A Force in Fragments: Reconstituting the Afghan National Army, Kabul, Brussels, Mai 2010, (Asia Report, 190), S. 1

26 James A. Schear/Wiliam B. Caldwell/Frank C. Digiovanni, »Developing Capacity for an Enduring Security Force«, in: PRISM, 2 (2011) 2, S. 135-144 (138).

27 Scott R. Lewis, »The Importance of Developing Government in Afghanistan «, in: Small Wars Journal, November 2011, S. 6 http://smallwarsjournal.com/ sites/default/files/lewis.pdf (18. Juli 2012).

28 Scott R. Lewis, »The Importance of Developing Government in Afghanistan «, in: Small Wars Journal, November 2011, S. 2; Terence Kelly/Nora Bensahel/Olga Oliker, Security Force Assistance in Afghanistan: Identifying Lessons for Future Efforts, Santa Monica, Ca.: Rand, 2011, S. 30.

29 Rebecca Barber, No Time to Lose. Promoting the Accountability of the Afghan National Security Forces, Oxford: Oxfam, Mai 2011, S. 31. 
Einflussnahme anfällig bleibt und in der Regel nur Armeeangehörige belangt werden, die nicht über Seilschaften verfügen. ${ }^{30}$

Die Entwicklungen ministerieller Fähigkeiten im Sicherheitsbereich fällt unter die Verantwortung des amerikanischen Combined Security Transition Command Afghanistan (CSTC-A), das wiederum über einen gemeinsamen Kommandeur mit der Trainingsmission der NATO verbunden ist (NTM-A). Dementsprechend stützte sich die Beratung der afghanischen Partner im Innen- und Verteidigungsministerium, bis zur Ebene des jeweiligen Ministers, stark auf Armeeoffiziere. Hier mangelte es lange Zeit an Beratern mit einem zivilen Hintergrund, insbesondere in den Bereichen Finanzverwaltung, Management, Politikformulierung und Logistik. Um diesem Problem entgegenzuwirken startete das amerikanische Verteidigungsministerium 2009 ein Programm zur Ausbildung und Entsendung von zivilem Fachpersonal in die afghanischen Sicherheitsministerien, das Ministry of Defense Advisor (MoDA)-Programm. ${ }^{31}$

Eine wesentliche Herausforderung besteht in Zukunft darin, den Aufbau ministerieller Kapazitäten im Sicherheitsbereich noch stärker als bisher in den breiteren Prozess der internationalen Bemühungen zur Stärkung guter Regierungsführung in Afghanistan einzubetten. Diese Aufgabe kann nicht allein von Beratern unter dem Kommando der US-Streitkräfte bzw. der NATO geleistet werden, so wichtig deren Beitrag auch ist.

Darüber hinaus müssen Maßnahmen zur Stärkung von Regierungsführung in dem Bewusstsein umgesetzt werden, dass in Afghanistan weiterhin ein Gegensatz zwischen formalen Institutionen, Gesetzen und Kodexen einerseits und informellen Machtstrukturen andererseits besteht. Entsprechende Maßnahmen dürfen sich daher nicht nur auf den Aufbau formaler Institutionen und die Verabschiedung von Gesetzen beschränken. Weitere Investitionen in die Verbesserung der Regierungsführung und den Aufbau einer funktionierenden Verwaltung im Sicherheitssektor müssen von deren tatsächlicher Umsetzung abhängig gemacht werden.

\section{Fazit: Widersprüche der internationalen Afghanistan-Politik}

Der Aufbau der afghanischen Sicherheitskräfte, der Umgang mit dem Problem der Milizen und die Bemühungen zum Aufbau einer funktionierenden Verwaltung im Sicherheitsbereich haben zentrale Widersprüchlichkeiten der internationalen Afghanistan-Politik deutlich gemacht. Die treibenden ausländischen Akteure der Transition - zuvorderst die USA und andere Truppensteller der ISAF bzw. deren Nachfolgemission ab 2015 sollten sich in den kommenden Jahren darauf konzentrieren, diese Widersprüche so weit wie möglich abzubauen.

Dabei geht es erstens um das Spannungsverhältnis zwischen den normativen Grundlagen der Transition, den gesellschaftlich-politischen Realitäten in Afghanistan und dem Handeln

\section{Ebd., S. 24}

31 James A. Schear/Wiliam B. Caldwell/Frank C. Digiovanni, "Developing Capacity for an Enduring Security Force , in: PRISM, 2 (2011) 2, S. 135-144 (136). ausländischer Akteure. Die internationale Politik war nach dem Sturz der Taliban 2001 eng mit dem Ziel verknüpft, in Afghanistan Sicherheitsinstitutionen als Teil einer umfassenden Statebuilding-Agenda aufzubauen.

Die Schwierigkeiten bei der Übertragung normativer Konzepte zur Reform des Sicherheitssektors (SSR) auf Afghanistan wurden in den NATO-Hauptstädten jedoch nur unzureichend thematisiert. So konnte die zivile Kontrolle des militärischen Apparats in Afghanistan bislang weder personell noch strukturell etabliert werden. Schlüsselpositionen in den Sicherheitsministerien werden weiterhin vor dem Hintergrund persönlicher und ethnischer Loyalitäten an ehemalige Kommandeure vergeben. Die internationalen Militärkräfte haben diese Entwicklungen zum Teil auch selbst mit zu verantworten, indem sie beispielsweise die formalisierten Kanäle in den afghanischen Ministerien umgangen haben. ${ }^{32}$

Unmittelbar damit hängt ein weiterer Widerspruch der internationalen Afghanistan-Politik zusammen. Dieser besteht zwischen den umfassenden zivil-militärischen Zielen der Transition und der lange einseitigen Fokussierung auf die Aufstellung und Bewaffnung von Polizei- und Militärkräften. Erst in jüngerer Zeit wurden auch die qualitativen Dimensionen des Aufbaus der afghanischen Sicherheitskräfte, der Verwaltungsaufbau bzw. die Regierungsführung sowie die Verknüpfung zwischen Polizei und Justiz stärker in den Blick genommen.

Drittens schließlich haben sich Widersprüchlichkeiten in der internationalen Afghanistan-Politik aus den konkurrierenden Zielsetzungen ergeben. Der NATO ist es bis heute nicht gelungen, das Handeln der ISAF in einen gemeinsamen strategischen Rahmen einzubetten, in dem Ziele und Prioritäten klarer als bisher definiert werden. Dies betrifft beispielsweise den ambivalenten Umgang mit Milizen und lokalen Sicherheitskräften, der den Aufbau eines Gewaltmonopols mit regulären Kräften untergraben kann. ${ }^{33}$

Das strategische Manko und die andauernd schlechte Sicherheitslage hatten zur Folge, dass die von den USA geführte internationale Koalition in Afghanistan gleichzeitig den SSRAnsatz verfolgte, militärische Aufstandsbekämpfung sowie Antiterrorpolitik betrieb und auf lokaler Ebene durch Adhoc-Vereinbarungen mit power brokern versuchte, Stabilität zu schaffen.

Da sich diese verschiedenen Ansätze zum Teil direkt widersprechen, führten sie im Endeffekt weder zu mehr Sicherheit noch zum Aufbau legitimer Sicherheitsorgane. ${ }^{34}$ Im Zuge der fortlaufenden Transition werden afghanische Regierung und ISAFTruppensteller die Prioritäten stärker auf den längerfristigen Aufbau des afghanischen Sicherheitssektors richten müssen.

32 Terence Kelly/Nora Bensahel/Olga Oliker, Security Force Assistance in Afghanistan: Identifying Lessons for Future Efforts, Santa Monica, Ca.: Rand, 2011, S. 75.

33 Philipp Münch, Strategielos in Afghanistan. Die Operationsführung der Bundeswehr im Rahmen der International Security Assistance Force, Berlin: Stiftung Wissenschaft und Politik, November 2011 (SWP-Studie 30/2011), S. 25.

34 Zur Widersprüchlichkeit der Zielsetzungen der internationalen AfghanistanPolitik vgl. Christian Dennys, Watching while the Frog Boils: Strategic Folly in the Afghan Security Sector, Waterloo: Centre for International Governance Innovation, Oktober 2011 (Afghanistan Papers, No. 9). 
Auch die nach 2014 von den USA geplanten Antiterror-Operationen sollten sich diesem Ziel unterordnen. ${ }^{35}$

Die genannten Widersprüche spiegeln auch reale Dilemmata wider - zwischen Wünschbarem und Machbarem, zwischen kurzfristigen und langfristigen Zielsetzungen - und lassen sich daher nicht in jedem Fall völlig auflösen. Die entscheidende Frage lautet jedoch, ob die „internationale Gemeinschaft“ den nötigen langen Atem haben wird, um die Transition tatsächlich „unumkehrbar“ zu machen.

Nach den Vorstellungen der Teilnehmer der internationalen Afghanistan-Konferenz, die im Dezember 2011 in Bonn unter Vorsitz des afghanischen Präsidenten stattfand, soll sich an das Ende der Transition 2014 eine „Transformationsdekade“ anschließen. Das Ziel lautet, Afghanistan während dieser Dekade zu einem voll funktionsfähigen Staat zu machen.

Auch die NATO bekannte sich während ihres Gipfeltreffens in Chicago im Mai 2012 erneut zu ihrer Verpflichtung auf ein langfristiges Engagement nach 2014. Flankierend dazu haben einzelne ISAF-Truppensteller, darunter die USA und Deutschland, bilaterale Abkommen mit der afghanischen Regierung abgeschlossen. Die Details des zukünftigen militärischen Engagements wurden jedoch auch in Chicago noch nicht festgelegt. Während der internationalen Geberkonferenz in Tokio, die Anfang Juli 2012 stattfand, wurden finanzielle Zusagen in Höhe von 16 Milliarden US-Dollar bis 2015 gemacht. ${ }^{36}$

Die Übergabe der Sicherheitsverantwortung an die nach wie vor schwache Zentralregierung in Kabul bleibt ein riskantes Unterfangen, zumal Pakistan weiterhin sichere Rückzugsgebiete für die Taliban bietet und eine Lösung zentraler Probleme des Landes - die Versöhnung der Bürgerkriegsparteien und die wirtschaftliche Entwicklung - höchst ungewiss bleibt. Ohne eine langfristig ausgerichtete Politik, die auch Lehren aus ihren Widersprüchen in der Vergangenheit zieht, wird die Übergabe der Sicherheitsverantwortung in Afghanistan nicht von Dauer sein.

\section{Nationale Identität, Gender und Gewaltbereitschaft}

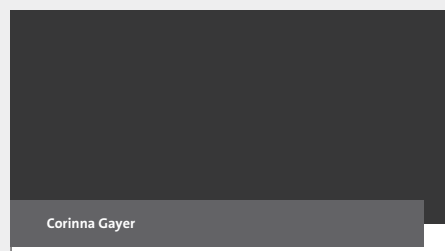

Gendered Intractability

National Identity Constructions and Gender in the Israeli-Palestinian Conflict

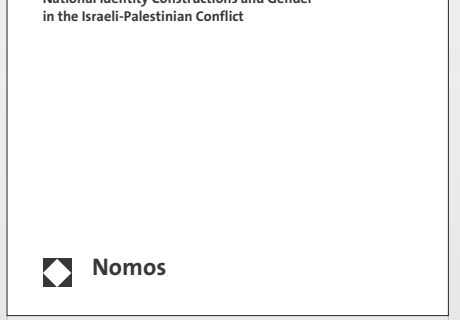

KY nowor

\author{
Gendered Intractability \\ National Identity Constructions and Gender in \\ the Israeli-Palestinian Conflict \\ Von Corinna Gayer \\ 2012, 346 S., brosch., 59,-€, ISBN 978-3-8329-7646-0
}

Diese Studie schlägt einen konzeptionell-methodologischen Rahmen zur Analyse nationaler Identitäten, Gender und der gewaltsamen Dynamik des israelischpalästinensischen Konflikts vor. Der Fokus liegt auf der Exploration der kognitiven, affektiven und konativen Aspekte nationaler Identitätskonstruktionen und ihren geschlechtsspezifischen Dimensionen und Implikationen.

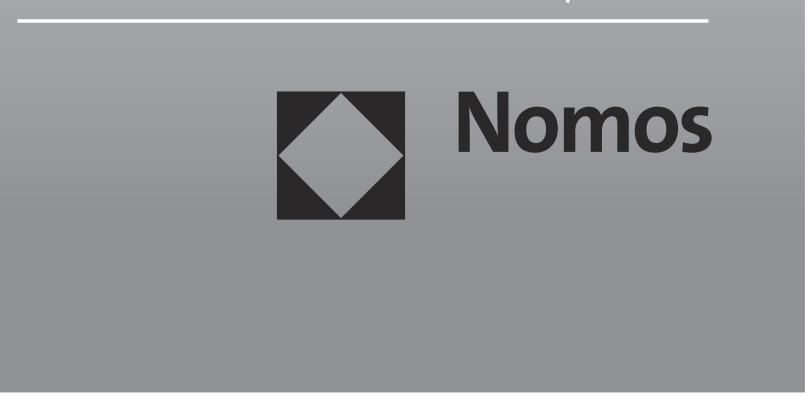

Nomos

Thomas Ruttig, einer der besten deutschen Afghanistan-Kenner, meint dazu: „Der Teilabzug [der Nato-Truppen] Ende 2014 ist [...] eher ein Formationswechsel, der das Gewicht derjenigen Einsatzkräfte stärkt, die am drastischsten für die gegenwärtige Polarisierung in Afghanistan verantwortlich sind."Thomas Ruttig, »Die Nato und ihr Bürgerkrieg«, in: tageszeitung, 22. Mai 2012.

36 Ministry of Foreign Affairs of Japan, Tokyo Conference on Afghanistan. The Tokyo Declaration. Partnership for Self-Reliance in Afghanistan. From Transition to Transformation, Tokio, 8. Juli 2012, http://www.mofa.go.jp/region/middle_e/ afghanistan/tokyo_conference_2012/tokyo_declaration_en1.html (10. Juli 2012). 